Following these hypotheses we developed a structured observation chart.

Intervention The chart we developed is contained on an A4 sheet. At the top there is a table with all the reasons for a baby requiring observations with the corresponding frequency of observation required. At the bottom there is a chart where the observations can be recorded. This incorporates a neonatal early warning system (NEWS) which can help to flag up early deterioration in patients (see inserted image).

Study design This audit was a prospective study. Over two weeks all babies on the two postnatal wards that required observations were analysed. The outcomes recorded were; the reason for the baby requiring observations and the frequency of observations carried out.

Strategy for change Currently the new observation chart is being approved by the care management group. After this has been done we will need to train the midwifes to use the chart, calculate the NEWS score and how to act on it. This will take around 6 months.

Measurement of improvement Once the chart has been implemented we will re-audit using the initial audit design. We will then compare the results to ascertain if the new chart has resulted in improvement in the frequency of observations.

Effects of changes The new observation chart has not yet been implemented into practice, however once it is in use it will be clearer how often observations need to be done and deterioration of a patient will be flagged up earlier therefore improving patient safety. A potential problem will be training the staff across sites to use the chart effectively.

Lessons learnt Awareness of the guidelines is key in ensuring their implemented. When it is not clear how often observations need to be done the frequency is well below the expected standard. However by implementing a chart that makes the frequency of observations clear and by combining a recording chart with NEWS it is hoped that recording of observation will dramatically increase and therefore improve patient safety.

Message for others As junior doctors it is key that when we spot something on the wards that affects patient safety we investigate its extent and root cause. We can then implement changes to improve patient safety. By using an observation chart which specifies the frequency of observation the number then missed will reduce, therefore any deterioration in a baby's observations will be spotted earlier. Additionally by using a NEWS the midwives will have a guide on how to act when a baby has abnormal observations. This again will help to identify unwell babies earlier.

\section{G578(P) POTENTIALLY PREVENTABLE UNEXPECTED TERM ADMISSIONS TO NEONATAL INTENSIVE CARE (NICU)}

${ }^{1} \mathrm{CL}$ Granger, ${ }^{2} \mathrm{~A}$ Okpapi, ${ }^{1} \mathrm{C}$ Peters, ${ }^{1} \mathrm{M}$ Campbell. ${ }^{1}$ Neonatal Intensive Care Unit, Royal Hospital for Sick Children, Glasgow, UK; ${ }^{2}$ Neonatal Intensive Care Unit, Wishaw General Hospital, Glasgow, UK

\subsection{6/archdischild-2015-308599.527}

Context/Problem Admission of babies to NICU for medical care involves the separation of mothers and babies. We questioned what proportion of term babies were admitted in our institution and what interventions they required. We hypothesised that a significant proportion of babies would have minimal intervention on admission and a number would have potentially avoidable and treatable causes that would lend themselves to quality improvement interventions. We aimed to identify causes of potentially preventable admissions as a key performance area to target.

Assessment of problem and analysis of cause We retrospectively reviewed the NICU database to identify all babies $\geq 37$ weeks gestation admitted to our tertiary level service over a 12-month period.

We classified term admissions as "expected" when NICU admission was anticipated following an antenatal diagnosis or "unexpected" where there were no concerns.

Study design Through Badgernet, demographic data were collected for all babies and the source of admission, diagnosis, interventions and length of stay (LoS) documented. For those infants in whom Badgernet data was incomplete or missing, retrospective case note analysis was undertaken.

Results There were 5843 babies delivered over the 12 month period of whom 4900 (83.9\%) were $\geq 37$ weeks. There were $453(9.2 \%)$ term babies admitted to NICU; 65 (14\%) of these admissions were expected and 389 (86\%) unexpected.

We identified a number of babies who did not meet the recommended standard for achieving high-level neonatal care benchmarked against admission temperature and blood sugar level. Blood sugar level was documented in 174 babies (44.7\%). Of these, 20 babies were identified as having a True Blood Glucose (TBG) $<2$ mmol. 25 babies had admission temperatures to the unit of $<36.5^{\circ} \mathrm{C}, 10$ with admission temperatures $<36^{\circ} \mathrm{C}$.

105 of 389 babies were discharged or transferred within $6 \mathrm{~h}$ of admission. 11/105 babies required medical intervention prior to transfer for cardiac or surgical management. The remaining 94 were discharged to the postnatal ward, 22 of 94 received IV antibiotics, no other interventions were required in the remaining 72.

$72 / 389(18.5 \%)$ of unexpected admissions at term required no medical interventions and were discharged to the postnatal ward within $6 \mathrm{~h}$, representing 9.5\% of all admissions to the unit.

Measurement of improvement Commonest reasons for admission in this group were mild respiratory distress, hypothermia and hypoglycaemia.

Following on from identifying these reversible causes we have implemented a quality improvement temperature bundle for use in labour ward, postnatal wards and NICU. This uses a visual cue to ensure both ambient temperature and infant temperatures are regularly checked. The introduction of this improvement bundle has enabled regular prospective audit of our temperature targets.

Conclusion and lessons learnt $9.2 \%$ of term infants were admitted to NICU. A significant proportion of "unexpected" admissions had a brief NICU stay and received minimal intervention. These infants who had minimal interventions represent a substantial share of the workload and admissions to NICU.

A concerning number of infants had hypothermia and hypoglycaemia on admission, a key area to target in implementation of quality improvement strategies.

Provision of simple supportive interventions in a Transitional Care Unit or observation area could potentially have reduced unexpected term admissions by $9.5 \%$.

\section{G579(P) PARENTAL PERCEPTION OF NEONATAL CARE}

VR Kistareddy, C Hauptfleisch, J McGowan. Neonatal Unit, NHS Grampian, Aberdeen, UK 
Context The improvement work was done in a neonatal unit inUK. It involved improvement in the care of newborns and included feedback from parents.

Problem The survey was done to assess parent's experiences of neonatal services, identify problems and get an understanding of how the quality of care can be improved. This helped us in developing a culture of continuous improvement that involves and is informed by parents, maintains high quality care for babies and their families and encourages the unit to continually raise the bar in provision of family-centred care.

Study design A questionnaire was sent to parents whose babies were discharged home over 4 months from December 2013 to March 2014. Parents were ineligible for inclusion if the care of the baby was re-oriented, if the baby had been taken into care or discharged to a post-natal ward. The Patient list was identified from Badger net. A Questionnaire and letter of introduction was prepared in consultation with medical and nursing staff. The questionnaire was sent by post once the baby had been discharged. Parents were reminded by phone a week later. Responses were returned to the neonatal secretaries by post.

Assessment of problem and analysis of its causes It was encouraging to see parents reporting positive experiences. There were problems which were identified from the feedback. The problems identified were parents were given inconsistent feeding plans and strategies from nursing staff, majority of parents of babies transferred to or from the unit felt the need to be with their baby during transfer, parents are asked to leave the ward during ward rounds to protect other babies' confidentiality and then usually relied on the nurses for feed back, it was felt by parents that the unit was outdated, overcrowded and stressful "not fit for purpose" and parents coming from outside the city felt the need for a day room and space for families. This will encourage their involvement in the care of their babies.

Strategy for change New NHS trust infant feeding policy and guidelines were formulated. Nursing staff were trained in regard to the policy. There is a need for a full-time breast feeding nurse, If possible, parents will accompany their baby on transfers, an improvement tree was placed in the unit for parents. This is easy to access and will help parents to comment on their experience and offer suggestions, parents will be encouraged to remain with their baby during ward rounds. This will help to involve them in the care of their baby and to update them, parents of babies who are likely to be in the unit for a prolonged time will also be given weekly updates by senior medical staff and plans for a new Women's hospital (2020) are underway and a parent focus group will be involved in the project to ensure their needs are met.

Measurement of improvement The survey will be repeated to see the effect of changes in strategy. Outcomes which have been encouraging have also been monitored by monitoring feeding policy and breastfeeding rates through Badger net and positive feedback from parent comments on the improvement tree.

Lessons learnt and message for others Most parents would like to be present during ward rounds; an opportunity to be updated by medical and nursing staff and allows them to be more involved in the day to day care of their baby; an improvement tree is a good way of getting feedback on a regular basis; Neonatal unit is a stressful environment and parents would benefit from a Day room and where possible, parents should be transported with their babies.

\section{G580(P) CHILD SAFEGUARDING CHECKLIST IN THE EMERGENCY CARE SETTING: IMPROVING CHECKLIST COMPLETION RATES}

S McLoughlin, S Khan. Emergency Care Centre, Royal Albert Edward Infirmary, Wigan, UK

10.1136/archdischild-2015-308599.529

Context This improvement work was done in the Paediatric Emergency Care Centre (PECC) of a busy district general hospital, which sees about 20,000 children every year. The work involved a collaboration of the nursing and medical staff in the PECC.

Problem When a child attends the PECC, a set of notes is generated which includes a Safeguarding Checklist on the second page. It is the responsibility of the clerking doctor to complete this for every child they see. It contains ten questions aimed at identifying children who could have experienced non-accidental injury (NAI). An audit showed that this checklist was only being completed $20 \%$ of the time. This was unacceptable as it put children at risk and contravened a basic trust policy.

Assessment of problem and analysis of its causes To establish why checklist completion was poor, a random sample of nonconsultant emergency doctors was given an anonymous questionnaire. The questionnaire, consisting of 5 multiple choice questions and a final open question, aimed to establish the barriers facing staff completing the checklist. A process map outlining a child's journey through the emergency floor and the above audit made it clear that the checklist was a barrier. Feedback indicated that the checklist itself was busy, unclear and difficult to use. As a result, I set about redesigning the checklist.

Intervention The Safeguarding Checklist now consists of 9 questions with the option to tick "yes", "no" or "not applicable" for each of them. Once they have been completed, there are instructions on how to categorise your findings into "green", "yellow" or "red", indicating the level of risk. Following this, there are bullet point directions of the next steps to take for each category.

Strategy for change The redesigned checklist was integrated in to the new PECC notes, replacing the previous version. The clerking clinicians complete the checklist but nursing staff assist in prompting them to do so. The questionnaire results were presented at a departmental meeting. Once the first draft of the redesigned checklist was completed, it was shown to PECC staff for feedback, which was incorporated in the final version.

Measurement of improvement Following the introduction of the new checklist, the notes of all children under five were audited five days a week to measure how often the checklist was being completed. Results after five months show it is being completed on average $65.6 \%$ of the time. Although this is an increase from $20 \%$, it still does not meet our aim of $100 \%$.

Effects of changes There is clearly an improvement in completion of the checklist but we aim to improve further. By increasing the use of the checklist, we hope to avoid further serious case reviews and promote a safeguarding culture within PECC.

It was difficult to bring together feedback from different sources to produce the final checklist. It was also challenging to create something that was simple and clear but still included all the important information.

Lessons learnt I learnt staff engagement, PDSA cycles and process mapping are key to making improvements. I also learnt that having an open and safe environment to express opinions leads to the some of the best suggestions. 